\title{
Discrimination of Quality and Geographical Origin of Extra Virgin Olive Oil by S3 Device with Metal Oxides Gas Sensors ${ }^{\dagger}$
}

\author{
Manohar P. Bhandari 1,2, , Estefanía Núñez Carmona 1, Marco Abbatangelo 1, \\ Veronica Sberveglieri ${ }^{3,4}$, Giorgio Duina ${ }^{1,4}$, Rajani Malla ${ }^{2}$, Elisabetta Comini ${ }^{1,4}$ and \\ Giorgio Sberveglieri ${ }^{1,4}$ \\ 1 Sensor Lab, Department of Information Engineering, University of Brescia, Via Branze 38, 25133 Brescia, \\ Italy; e.nunezcarmona@unibs.it (E.N.C.); m.abbatangelo@unibs.it (M.A.); giorgio.duina@nasys.it (G.D.); \\ elisabetta.comini@unibs.it (E.C.); giorgio.sberveglieri@unibs.it (G.S.) \\ 2 Central Department of Biotechnology, Tribhuvan University, 44613 Kirtipur, Nepal; \\ rajanimalla2000@gmail.com \\ 3 CNR-IBBR, Via Madonna del Piano 10, 50019 Sesto Fiorentino, Florence, Italy; \\ veronica.sberveglieri@ibbr.cnr.it \\ 4 Nano Sensor Systems s.r.l., Via Branze 38, 25123 Brescia, Italy \\ * Correspondence: m.bhandari@unibs.it; Tel.: +39-388-880-9842 \\ † Presented at the Eurosensors 2018 Conference, Graz, Austria, 9-12 September 2018.
}

Published: 26 November 2018

\begin{abstract}
In the present work, a gas sensor device S3 based on an array of eight metal oxides semiconductor gas sensors has been demonstrated and applied to the discrimination of quality and geographical origins of the Italian extra virgin olive oils. Furthermore, the principal component analysis (PCA) and artificial neural networks (ANN) were carried out on the set of data acquired from the sensor array response to the extra virgin olive oil headspace. The preliminary results have shown a good capability of the instrument to distinguish different kind of extra virgin olive oil samples and thus evaluate their quality and origin.
\end{abstract}

Keywords: metal oxides; nanowire gas sensors; gas sensor device; electronic nose; extra virgin olive oil; olive oil quality; geographical origin

\section{Introduction}

Extra virgin olive oil (EVOO) is one of the most commonly adulterated food products because of its high price, health benefits, and nutritive values. The quality and authenticity of an EVOO are strongly related to its aroma, organoleptic properties, cultivar, and geographical origin. Moreover, the European Commission has introduced a legislation in order to safeguard the Protected Designation of Origin (PDO) trademark of some EVOOs linked with the production area [1]. For this reason, it is important to monitor and ensure the quality and geographical origin of the EVOO $[2,3]$.

The gas sensor devices or electronic noses based on the metal oxides semiconductor gas sensors are analytical and sensory instruments particularly useful in the comparative evaluation of the quality of EVOO [4]. The chemical sensors employed within an instrument can determine the volatile organic compounds emitted by olive oils to detect fraud and assess the quality of the product. The aim of this work is to apply a versatile metal oxide nanowires gas sensors-based device S3 in the discrimination of commercial EVOO samples with different qualities and geographical origin. Afterwards, the data obtained from the sensor array response were analyzed using PCA and ANN for the rapid pattern screening of the olive oil samples. 


\section{Materials and Methods}

\subsection{S3 Device and Gas Sensors Used}

Herein, we report a state-of-the-art metal oxide nanowires gas sensor device S3 (Nano Sensor Systems srl, Brescia, Italy) for EVOO analysis. S3 system comprises of a pneumatic part, an electronic part, an incubated sensor chamber, and an online data acquisition and processing app. The sensing elements used were eight metal oxides gas sensors and one temperature/humidity sensor. Three of the sensors in the array were tin oxide and copper oxide nanowire gas sensors (two $\mathrm{SnO}_{2}-\mathrm{Au}$, one $\mathrm{CuO}$ ), three RGTO thin film tin oxide gas sensors (two $\mathrm{SnO}_{2}$, one $\mathrm{SnO}_{2}$ catalyzed with $\mathrm{Au}$ ), and two Figaro TGS sensors (TGS2600, TGS2602). The details of the gas sensors integrated in S3 are summarized in Table 1.

Table 1. Sensors type, composition, morphology, and operating temperature $\left({ }^{\circ} \mathrm{C}\right)$ used in S3 device.

\begin{tabular}{cccc}
\hline $\begin{array}{c}\text { Sensor Material } \\
\text { (Type) }\end{array}$ & Composition & Morphology & $\begin{array}{c}\text { Operating } \\
\text { Temperature }\left({ }^{\circ} \mathbf{C}\right)\end{array}$ \\
\hline $\mathrm{SnO}_{2}-\mathrm{Au}(\mathrm{n})$ & $\mathrm{SnO}_{2}$ grown with $\mathrm{Au}$ & Nanowire & 350 \\
$\mathrm{SnO}_{2}-\mathrm{Au}(\mathrm{n})$ & $\mathrm{SnO}_{2}$ grown with $\mathrm{Au}$ & Nanowire & 400 \\
$\mathrm{CuO}(\mathrm{p})$ & $\mathrm{CuO}$ & Nanowire & 400 \\
$\mathrm{SnO}_{2}(\mathrm{n})$ & $\mathrm{SnO}_{2}$ & $\mathrm{RGTO}$ & 300 \\
$\mathrm{SnO}_{2}(\mathrm{n})$ & $\mathrm{SnO}_{2}$ & RGTO & 400 \\
$\mathrm{SnO}_{2}-\mathrm{Au}+\mathrm{Au}(\mathrm{n})$ & $\mathrm{SnO}_{2}$ grown with Au and & RGTO & 350 \\
\hline
\end{tabular}

The complex set of data acquired from the $\mathrm{S} 3$ sensors response to the volatile organic compounds originating from the EVOO headspace were analyzed through a multivariate analysis PCA and ANN. The measurements were performed in an autosampler HT2800T (HTA srl, Brescia, Italy) [5,6]. For this, $5 \mathrm{~mL}$ of olive oil samples were enclosed in $20 \mathrm{~mL}$ vials and placed randomly in the autosampler. The sample headspace was drawn out from the vial and injected at $1 \mathrm{~mL} / \mathrm{min}$ into a chromatographic air flow $(50 \mathrm{sccm})$. The injection time was $2 \mathrm{~min}$ and the recovery time was $5 \mathrm{~min}$.

\subsection{Olive Oil Samples}

A total of seven commercial olive oil samples obtained from a local supermarket were analyzed and classified using the S3. Six of them were EVOOs and the remaining was an olive oil or lampante (Table 2). The two cultivars of the Garda DOP EVOOs coming from the east and the west shores of Lake Garda in north Italy were analyzed to differentiate their latitude of production.

Table 2. Description of olive oil samples in relation to type, provenience zone, harvested year, and processing method.

\begin{tabular}{cccc}
\hline Olive Oil Type & Provenience Zone & Harvested Year & Processing Method \\
\hline Garda DOP East EVOO & Italy & $2016 / 2017$ & Filtered \\
Garda DOP West EVOO & Italy & $2016 / 2017$ & Cold Extraction, Filtered \\
EVOO & Italy & 2016 & Filtered \\
Organic EVOO & Italy & 2017 & Filtered \\
EVOO & Italy & 2016 & Cold Extraction, Filtered \\
EVOO & Italy & 2016 & Cold Extraction, Unfiltered \\
Olive Oil & Italy & 2017 & Filtered \\
\hline
\end{tabular}

\section{Results and Discussion}

The PCA score plot shown in Figure 1 reports the separation among the EVOO samples in relation to the quality, cultivar, and processing method. For the $\mathrm{S} 3$ analysis, the resistances of the gas sensors were taken into consideration and normalized prior to PCA and ANN classification. When 
extracting five features and taking the minimum value of sensor resistance, a clear discrimination was found between all the EVOOs and the olive oil. Similarly, a good distinction between the unfiltered and the filtered EVOOs was obtained.

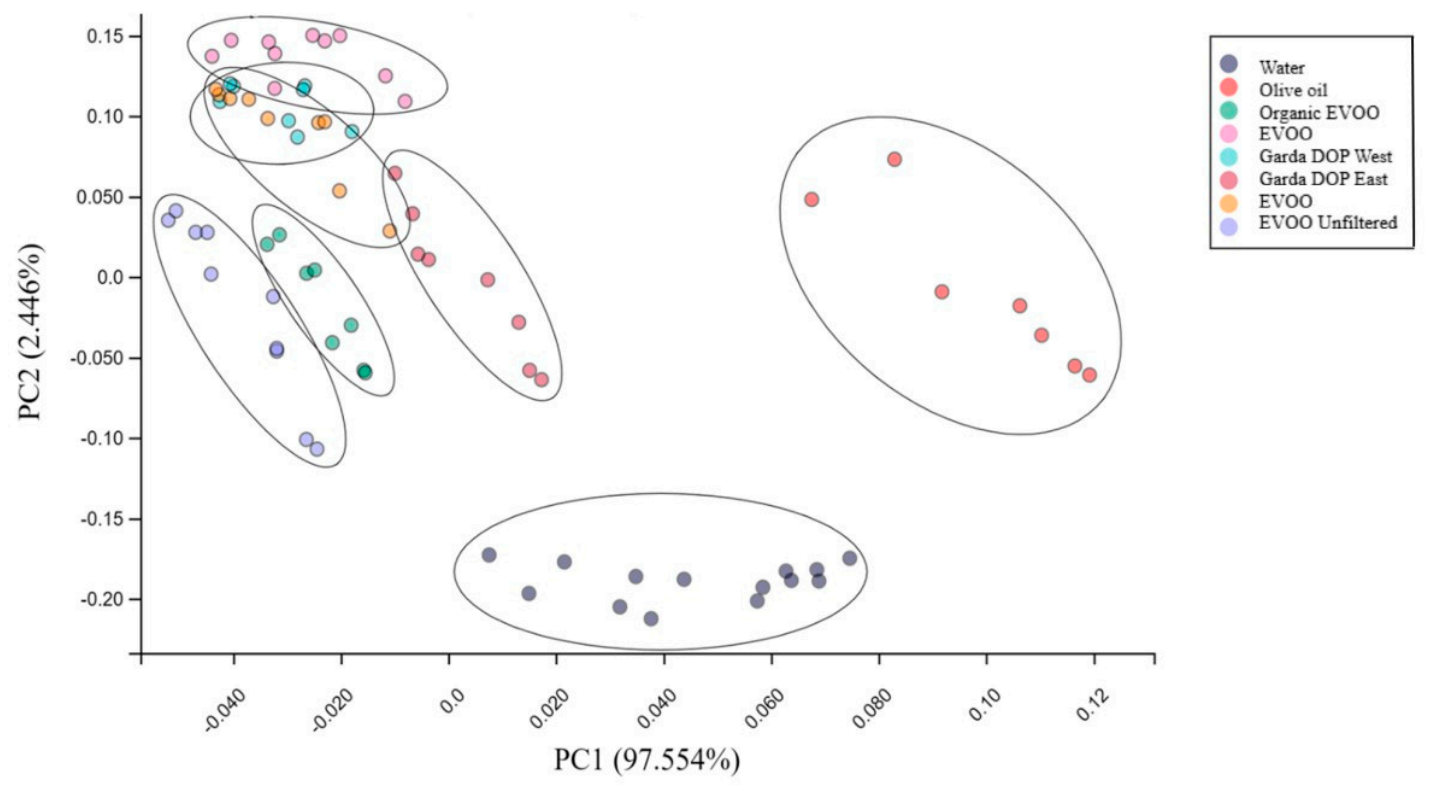

Figure 1. PCA score plot (PC1 vs. PC2) of eight metal oxides gas sensors response with respect to the target category olive oil samples and water (as control) represented by different clusters. Total explained variance: $\mathrm{PC} 1=97.554 \%, \mathrm{PC} 2=2.446 \%$.

In Figure 2, a PCA score plot for the geographical origin of two different Garda DOP EVOOs is shown. Through two variables PC1 and PC2, the two varieties of the Garda DOP EVOOs produced in the east and the west shores of Lake Garda were distinctively separated as seen in the figure. Moreover, the two variables explained almost all information resulting in a good classification. Thus, the S3 device was able to analyze the volatile fraction of substances present in the two Garda DOP EVOOs headspace in a rapid and reproducibleway.

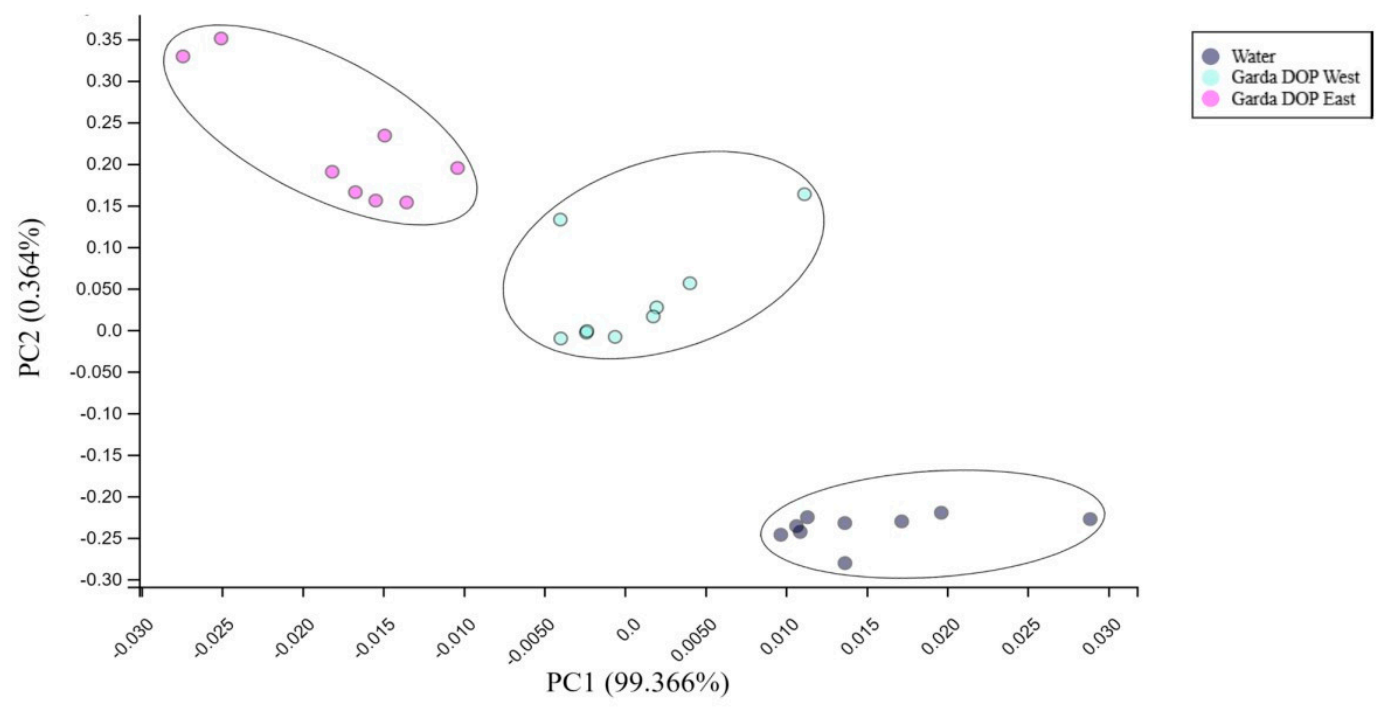

Figure 2. PCA score plot (PC1 vs. PC2) of eight metal oxides gas sensors performed on the Garda DOP East and the Garda DOP West EVOO samples represented by two different clusters. Total explained variance: $\mathrm{PC} 1=99.366 \%, \mathrm{PC} 2=0.364 \%$. 


\section{Conclusions}

In conclusion, the results herein reported verify that the novel metal oxides gas sensor device S3 represents a very useful handheld, fast, and non-invasive tool for the on-line monitoring and evaluation of the quality and geographical origin of extra virgin olive oils. This also inspires the use of the S3 device in EVOO fraud detection and authenticity control in general. The multivariate statistical analysis involving the PCA and ANN highlights the highest classification rates that will be useful to have greater statistical significance of the future results.

Acknowledgments: M.P.B. is grateful to the Erasmus Mundus LEADER Project, Action 2, Strand 1 funded by the European Audiovisual and Culture Executive Agency of the European Commission.

Conflicts of Interest: The authors declare no conflict of interest.

\section{References}

1. Council of the European Union. Council Regulation (EEC) No 2081/92; 1992; pp. 1-8. Available online: https://eur-lex.europa.eu/legal-content/EN/TXT/?uri=celex\%3A31992R2081 (accessed on 22 November 2018).

2. Haddi, Z.; Alami, H.; El Bari, N.; Tounsi, M.; Barhoumi, H.; Maaref, A.; Jaffrezic-Renault, N.; Bouchikhi, B. Electronic nose and tongue combination for improved classification of Moroccan virgin olive oil profiles. Food Res. Int. 2013, 54, 1488-1498, doi:10.1016/j.foodres.2013.09.036.

3. Cosio, M.S.; Ballabio, D.; Benedetti, S.; Gigliotti, C. Geographical origin and authentication of extra virgin olive oils by an electronic nose in combination with artificial neural networks. Anal. Chim. Acta 2006, 567, 202-210, doi:10.1016/j.aca.2006.03.035.

4. García-González, D.L.; Aparicio, R. Virgin olive oil quality classification combining neural network and MOS sensors. J. Agric. Food Chem. 2003, 51, 3515-3519, doi:10.1021/jf021217a.

5. Sberveglieri, V.; Bhandari, M.P.; Núñez Carmona, E.; Betto, G.; Soprani, M.; Malla, R.; Sberveglieri, G. Spectrocolorimetry and nanowire gas sensor device S3 for the analysis of Parmigiano Reggiano cheese ripening. In Proceedings of the 2017 ISOCS/IEEE International Symposium on Olfaction and Electronic Nose (ISOEN), Montreal, QC, Canada, 28-31 May 2017; doi:10.1109/ISOEN.2017.7968861.

6. Abbatangelo, M.; Núñez Carmona, E.; Sberveglieri, V.; Zappa, D.; Comini, E.; Sberveglieri, G. Application of a Novel S3 Nanowire Gas Sensor Device in Parallel with GC-MS for the Identification of Rind Percentage of Grated Parmigiano Reggiano. Sensors 2018, 18, 1617, doi:10.3390/s18051617.

(C) 2018 by the authors. Licensee MDPI, Basel, Switzerland. This article is an open access article distributed under the terms and conditions of the Creative Commons Attribution (CC BY) license (http://creativecommons.org/licenses/by/4.0/). 